\title{
Prime Fano threefolds and integrable systems
}

\author{
A. Iliev, L. Manivel
}

\begin{abstract}
For a general K3 surface $S$ of genus $g, 2 \leq g \leq 10$, we prove that the intermediate Jacobians of the family of prime Fano threefolds of genus $g$ containing $S$ as a hyperplane section, form generically an algebraic completely integrable system.
\end{abstract}

\section{Introduction}

The punctual Hilbert schemes $H i l b_{d} S$ of length $d$ subschemes of a smooth K3 surface $S$ are examples of smooth complex symplectic varieties Be1. The K3 surfaces are the only smooth compact complex symplectic varieties of dimension two. Any curve $C$ on a K3 surface is automatically a Lagrangian subvariety of $S$, and if $C \subset S$ is a smooth curve of genus $g$ then the relative Jacobian $\mathcal{J}$ is a Lagrangian fibration (equivalently, an algebraically completely integrable system, see $[\mathrm{DM}, 2.3)$ over an open subset of the complete linear system $|C| \simeq \mathbb{P}^{g}$, see $[\mathrm{Be} 2$. In fact, if all the curves in $|C|$ are reduced, then the compactified relative Jacobian $\overline{\mathcal{J}} \rightarrow|C| \simeq \mathbb{P}^{g}$ is a smooth symplectic variety and still remains a Lagrangian fibration, see also $\S 4$ in Bot. Recently Sawon, by using the techniques of the Fourier-Mukai functors, managed to show that for any $k \geq 2$, and for the general K3 surface $S$ with primitive polarization of degree $d=k^{2}(2 g-2)$, the Hilbert scheme Hilb $_{g} S$ is locally isomorphic to a compactified relative Jacobian $\overline{\mathcal{J}} \rightarrow \mathbb{P}^{g}$ as above, thus proving the existence of a Lagrangian fibration on $\mathrm{Hilb}_{g} S$ over $\mathbb{P}^{g}$, see $[\mathrm{Saw}$. The fibrations of Beauville and Sawon are fibered generically by Jacobians of curves, and one may ask how to find other types of Lagrangian fibrations.

A way towards new examples is the following criterion of integrability, due to Donagi and Markman and generalizing the above construction of Beauville (see 8.1-8.2 in [DM]):

(DM) Let $M$ be a smooth complex symplectic variety, and let $Z \subset M$ be a smooth Lagrangian subvariety. Then the relative Picard bundle Pic ${ }^{O}$ is a Lagrangian fibration (an algebraically completely integrable system) over an open set of the base of deformations of $Z$ in $M$.

In general, the criterion (DM) raises the problem of finding examples of complex symplectic varieties $M$ (different from K3 surfaces) and Lagrangian subvarieties $Z$ with well understood Picard variety and deformations in $M$.

The first higher dimensional symplectic variety that has been exploited in this context is the Beauville-Donagi variety - the Fano fourfold $F(W)$ of lines on the general smooth cubic fourfold $W$, see $[\mathrm{BD}$. This is performed in two different setups, corresponding to two basic families of Lagrangian surfaces in $F(W)$ arising from the geometry of cubic hypersurfaces of

\footnotetext{
${ }^{1}$ MSC 14J28, 14J45, 14K30, 37J99

2 The 1-st author is partially supported by grant MI1503/2005 of the Bulgarian Foundation for Scientific Research
} 
dimensions 3 and 5. In the first case, as observed by Voisin, the Fano families of lines on the general cubic threefolds contained in $W$ as hyperplane sections are smooth Lagrangian surfaces in $F(W)$, see Exemple 3 in [Voi]. Applying (DM), one obtains (see Example 8.22 of [DM]): The relative intermediate Jacobian over the family of smooth cubic threefolds, contained as hyperplane sections of $W$, is an algebraic integrable system. In the second case, by our recent observation (see [IM2]) the Fano families of planes on the general cubic fivefolds containing $W$ as a hyperplane section are embedded by the intersection map as smooth Lagrangian surfaces in $F(W)$. This yields the second application of (DM), which is in some sense symmetric to the first: The relative intermediate Jacobian over the family of smooth cubic fivefolds, containing $W$ as a hyperplane section, is an algebraic integrable system, ibid.

In the present paper we consider, instead of the Beauville-Donagi variety, the Hilbert powers $H_{i l b} S$ of smooth K3 surfaces $S$ contained in prime Fano threefolds.

Recall that a prime Fano threefold is a smooth compact complex variety $X$ of dimension 3 for which the anticanonical divisor $H=-K_{X}$ is the ample generator of $\operatorname{Pic}(X)$. For such $X$, the number $g=-K_{X}^{3} / 2+1$ is always a positive integer, called the genus of $X=X_{2 g-2}$. Prime Fano threefolds exist only for $2 \leq g \leq 12, g \neq 11$ (see [IP]). A general member of the anticanonical linear system $\left|-K_{X}\right|$ of a prime Fano threefold $X=X_{2 g-2}$, is a K3 surface $S=S_{2 g-2}$ of genus $g$. Mukai proved that the converse is also true: the general K3 surface of genus $g, 2 \leq g \leq 12, g \neq 11$ can be embedded as an anticanonical divisor in a prime Fano threefold of genus $g$, see [Muk1] or [Be3] for a more precise statement. In this situation takes place the following observation, due originally to Thomas (see Theorem 1.6.1 in [Tho]):

Let $S=S_{2 g-2}$ be a K3 surface in a prime Fano threefold $X=X_{2 g-2}$ of genus $g$. Then for any integer $d \geq 1$, any component $\mathcal{H}$ of the Hilbert scheme of smooth curves of degree $d$ on $X$ is sent by the intersection map $j$ with $S$ to a Lagrangian subvariety $j(\mathcal{H})$ of $\mathrm{Hilb}_{d} S$,

see Lemma 1. Since our study will require a closer inspection of the intersection map $j$, in $\S 2$ we write down a detailed proof of Lemma 1. In particular, we state a criterion for $j$ to be an immersion (see Corollary 2).

In $\S 3$ and $\S 4.1$ we study the scheme $F(X)$ of conics on a prime Fano threefold $X=X_{2 g-2}$. Notice that together with the family of lines, this is one of the two best studied families of curves on prime Fano threefolds; see Ch.4 of IP for a survey of the basic results about lines and conics on Fano threefolds. In $\S 3.1$ we collect the most important, at least from our perspective, of these results, beginning with the fact that for $X$ general, $F(X)$ is a smooth irreducible surface, the Fano surface of $X$. In $\S 3.2$ we study the intersection map $j: F(X) \rightarrow \mathrm{Hilb}_{2} S$ for the general pair $(X, S)$ of a prime Fano threefold $X$ and a K3 surface $S \in\left|-K_{X}\right|$. By Lemma $1, j(F(X))$ is a Lagrangian surface in the symplectic fourfold $\mathrm{Hilb}_{2} \mathrm{~S}$.

In order to apply the integrability criterion (DM), we need to know whether $j(F(X))$ is smooth. By the Proof of Proposition 4. for $g \geq 7$ the map $j$ is injective; in particular the isomorphic image $j(F(X))$ of the smooth Fano surface $F(X)$ is a smooth Lagrangian subvariety of $\mathrm{Hilb}_{2} \mathrm{~S}$. So for $g \geq 7$ we can directly apply the criterion (DM) to get an integrable system. However for $g \leq 6$ the image $j(F(X))$ is not smooth, even for the general $X$, and the criterion (DM) cannot be directly applied to pairs $(Z, M)=\left(j(F(X)), \mathrm{Hilb}_{2} S\right)$. More concretely, for any $g \leq 6$ the map $j$ is almost an isomorphism from $F(X)$ onto its image, except for a finite number of multiple points (presumably simple double points).

Nevertheless, by Proposition 4 for the general pair $(X, S)$, of a prime Fano threefold $X$ of any possible genus $g$ and a K3 surface $S \in\left|-K_{X}\right|$ the intersection map $j: F(X) \rightarrow \mathrm{Hilb}_{2} S$ is 
in fact an immersion. In this situation, due to results of Ran and Voisin, we still can apply the criterion (DM), by replacing in its conditions the smooth Lagrangian subvariety by a Lagrangian immersion of a smooth variety, see $\S 4.2$. This gives rise from $j(F(X)) \subset \mathrm{Hilb}_{2} S$ to an algebraic integrable system (i.e. a Lagrangian fibration) - the relative Picard $P i c^{o} \rightarrow \mathcal{H}$ over the base $\mathcal{H}$ of the deformation space of $j$ (with a fixed target), see $\S 4.2$.

It turns out that we can exactly describe the fibers of this Lagrangian fibration. We make this in two steps. In general, the fibers of such a relative Picard are the Picard schemes of certain smooth surfaces $F_{s}$, obtained as deformation of a fixed $F(X)=F_{o}$. In our particular case, Proposition 9 shows that all these surfaces $F_{s}$ are in fact Fano surfaces of Fano threefolds $X_{s}$ containing the K3 surface $S$, at least in a neighbourhood of $F(X)$. Next, due to a less known but general fact about prime Fano threefolds, the Abel-Jacobi map from the Albanese variety Alb $F(X)$ of $F(X)$ to the intermediate Jacobian $J(X)$ of $X$ is an isomorphism, at least for the general prime $X$ of any genus $g$, see Theorem 8 . Now by Theorem 8 , and by the fact that the intermediate Jacobians $J\left(X_{s}\right)$ are principally polarized abelian varieties, $J\left(X_{s}\right) \simeq \operatorname{Alb} F\left(X_{s}\right) \simeq$ $P i c^{o} F\left(X_{s}\right)$. This yields the main result of the paper, Theorem 10

For a general $K 3$ surface $S$ of genus $2 \leq g \leq 10$, the relative intermediate Jacobian is generically an algebraic integrable system over the family of prime Fano threefolds containing S.

There is a strong analogy with the fact that the relative intermediate Jacobian is generically a completely integrable system over a complete deformation of gauged Calabi-Yau threefolds (DM], Theorem 7.7). Here we considered families of $\log$ Calabi-Yau varieties with a fixed boundary, that is, families of pairs $(X, S)$ with $X$ a (Fano) threefold and $S$ a divisor such that $K_{X}+S=0$ (the gauge disappears). The boundary $S$ is our fixed K3 surface. It would be interesting to extend our main result to more general families of log Calabi-Yau threefolds.

\section{Lagrangian images of families of curves on Fano threefolds}

Recall from [Muk1] and [Be3] that for any $2 \leq g \leq 12, g \neq 11$, the general K3 surface $S=S_{2 g-2}$ of genus $g$ can be represented as an anticanonical divisor in a Fano threefold $X=X_{2 g-2}$ of genus $g$. For $g \geq 3$, the anticanonical bundle $-K_{X}$ is very ample, so $S$ is just a hyperplane section in the anticanonical embedding of $X$.

Lemma 1 Let $X=X_{2 g-2}$ be a Fano threefold of genus $g, 2 \leq g \leq 12, g \neq 11$, and let $S$ be a general member of the anticanonical system $\left|-K_{X}\right|$. Let $\mathcal{H}$ be an irreducible component of the Hilbert scheme of curves on $X$, whose general member is a smooth curve of anticanonical degree $d$, and consider the intersection map

$$
j: \mathcal{H} \rightarrow \mathrm{Hilb}_{d} S, \quad j: C \mapsto C \cap S .
$$

Then the image $j(\mathcal{H}) \subset H i l b_{d} S$ is a Lagrangian subvariety of $H i l b_{d} S$.

Proof. Let the curve $C \subset X$ be a general element of $\mathcal{H}$. Since the anticanonical divisor is basepoint free, and $S \in\left|-K_{X}\right|$ is also general, the intersection $C \cap S=\left\{x_{1}, \ldots, x_{d}\right\}$ is a reduced zero-scheme of length $d$. Consider the differential

$$
d_{C} j: T_{C} \mathcal{H}=H^{0}\left(N_{C / X}\right) \longrightarrow T_{C \cap S} H i l b_{d} S=T_{x_{1}} S \oplus \cdots \oplus T_{x_{d}} S .
$$


The Hilbert scheme $\mathrm{Hilb}_{d} S$ has a symplectic structure

$$
\wedge^{2} T_{C \cap S} H i l b_{2} S \longrightarrow \wedge^{2} T_{x_{1}} S \oplus \cdots \oplus \wedge^{2} T_{x_{d}} S \stackrel{\sigma_{x_{1}}, \ldots, \sigma_{x_{d}}}{\longrightarrow} \mathbb{C} \oplus \cdots \oplus \mathbb{C} \stackrel{+}{\longrightarrow} \mathbb{C}
$$

defined by the choice of a non zero section $\sigma \in H^{0}\left(S, K_{S}\right)=H^{0}\left(S, \Omega_{S}^{2}\right) \simeq \mathbb{C}$.

Denote by $j_{x}$ the skew-symmetric form defined by the composition

$$
\wedge^{2} H^{0}\left(N_{C / X}\right) \rightarrow \wedge^{2} T_{x} S \stackrel{\sigma_{x}}{\rightarrow} \mathbb{C} .
$$

We shall see first that $j_{x_{1}}+\cdots+j_{x_{d}}=0$, i.e. that $j(\mathcal{H})$ is an isotropic subvariety of $H i l b_{d} S$ at the point $j(C)=x_{1}+\cdots+x_{d}$ with respect to the symplectic structure on $H i l b_{d} S$. For this, consider the diagram

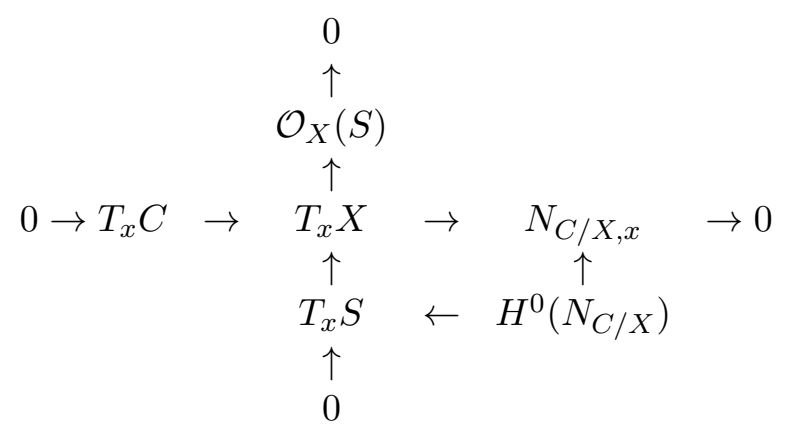

The vertical sequence gives a natural isomorphism $K_{X}(S)_{\mid S}=K_{S}$ (residue). The horizontal sequence gives $K_{X \mid C}=K_{C} \otimes\left(\operatorname{det} N_{C / X}\right)^{*}$. So $\sigma$ lifts uniquely to a section of $K_{X}(S)$, a meromorphic 3 -form on $X$ with poles on $S$. By restriction to $C$, we get a section $\tilde{\sigma}$ of $K_{C}(C \cap S) \otimes\left(\operatorname{det} N_{C / X}\right)^{*}$. Let $\alpha, \beta$ be global sections of $N_{C / X}$. Then $\alpha \wedge \beta$ is a global section of $\operatorname{det} N_{C / X}$, and its product with $\tilde{\sigma}$ gives a section $(\sigma, \alpha \wedge \beta)$ of $K_{C}(C \cap S)$, a meromorphic form on $C$ with poles at $x_{1}, \ldots, x_{d}$. Now, for any $x \in S$ the following identity takes place

$$
j_{x}(\alpha, \beta)=\operatorname{res}_{x}(\sigma, \alpha \wedge \beta) .
$$

To verify $(*)$, choose local coordinates $u, v, w$ on $X$ around $x$, such that $u, v$ are local equations of $C$ and $w$ a local equation of $S$. Our generator $\sigma$ of $K_{S}$ can be written locally as $f d u \wedge d v$ for $f$ some regular function on $S$, and it is the residue of the form $\tilde{\sigma}$ on $X$ which is given locally by

$$
\tilde{\sigma}=F d u \wedge d v \wedge \frac{d w}{w}, \quad F_{\mid S}=f
$$

Since $\partial / \partial u$ and $\partial / \partial v$ are local generators of $N_{C / X}$, we write locally $\alpha \wedge \beta$ as $a \partial / \partial u \wedge \partial / \partial v$. The result of the contraction with the restriction of $\tilde{\sigma}$ to $C$ is $(\sigma, \alpha \wedge \beta)=F_{\mid C} a \frac{d w}{w}$, whose residue at $x$ is $\operatorname{res}_{x}(\sigma, \alpha \wedge \beta)=F(x) a(x)=f(x) a(x)$. On the other hand, $j_{x}(\alpha, \beta)$ is obtained by evaluating $\sigma_{x}$ on $a(x) \partial / \partial u \wedge \partial / \partial v$ considered as a vector of $\wedge^{2} T_{x} S$. This gives $j_{x}(\alpha, \beta)=f(x) a(x)$, as claimed.

Next, by $\left(^{*}\right)$ and the residue theorem,

$$
j_{x_{1}}(\alpha, \beta)+\cdots+j_{x_{d}}(\alpha, \beta)=\operatorname{res}_{x_{1}}(\sigma, \alpha \wedge \beta)+\cdots+\operatorname{res}_{x_{d}}(\sigma, \alpha \wedge \beta)=0,
$$

which proves that $j(\mathcal{H})$ is an isotropic subvariety of $H i l b_{d} S$ at $x_{1}+\cdots+x_{d}$. 
Since $x_{1}+\cdot+x_{d}$ as above is a general point of $j(\mathcal{H})$, then $j(\mathcal{H})$ is an isotropic subvariety of $H i l b_{d} S$; and in order to prove that $j(\mathcal{H})$ is a Lagrangian subvariety of $H i l b_{d} S$ it remains to see that $j(\mathcal{H})$ has dimension $d$.

For this, consider the differential of $j$ at a general member $C$ of the family $\mathcal{H}$.

Let $I_{C}$ and $N=N_{C / X}$ denote the ideal sheaf and the normal sheaf of $C$ in $X$. Since the general $C \in \mathcal{H}$ is smooth, in particular $C$ is a locally complete intersection in $X$, then $N_{C / X}$ is locally free. Let

$$
T_{C} \mathcal{H}=H^{0}(N)=\operatorname{Hom}\left(I_{C} /\left(I_{C}\right)^{2}, \mathcal{O}_{C}\right) \stackrel{d_{C j}}{\longrightarrow} T_{C \cap S} H i l b_{d} S=\operatorname{Hom}\left(I_{C \cap S}^{S} /\left(I_{C \cap S}^{S}\right)^{2}, \mathcal{O}_{C \cap S}\right)
$$

be the natural map deduced from the identity $I_{C \cap S}^{S}=\left(I_{C}+I_{S}\right) / I_{S}$ for the ideal sheaf of $C \cap S$ in $S$, and let

$$
T_{C \cap S} H_{i l b} S=\operatorname{Hom}\left(I_{C \cap S}^{S} /\left(I_{C \cap S}^{S}\right)^{2}, \mathcal{O}_{C \cap S}\right) \stackrel{\theta}{\longrightarrow} H o m\left(I_{C} /\left(I_{C}\right)^{2}, \mathcal{O}_{C \cap S}\right)=N \otimes_{\mathcal{O}_{X}} \mathcal{O}_{C \cap S}
$$

be the map deduced from the restriction $I_{C} \rightarrow I_{C \cap S}^{S}$. Since this restriction map is surjective, $\theta$ is injective. Moreover, the composition

$$
H^{0}(N) \stackrel{\theta \circ d_{C} j}{\longrightarrow} N \otimes \mathcal{O}_{X} \mathcal{O}_{C \cap S}
$$

is just the evaluation map $e v_{C \cap S}$ on the punctual scheme $C \cap S$. In particular, the kernel of $d_{C} j$ coincides with the kernel of $e v_{C \cap S}$, which can easily be computed from the exact sequence $0 \rightarrow \mathcal{O}_{C}(-S) \rightarrow \mathcal{O}_{C} \rightarrow \mathcal{O}_{C \cap S} \rightarrow 0$, twisted by the locally free sheaf $N$. We get

$$
\operatorname{Ker} d_{C} j=H^{0}(N(-S))=H^{0}\left(N \otimes K_{X \mid C}\right)=H^{0}\left(N^{*} \otimes K_{C}\right) \simeq H^{1}(N)^{*} .
$$

This implies that the relative dimension of $j$ is $h^{1}(N)$. Then, if $g$ denotes the arithmetic genus of $C$, the Riemann-Roch yields

$$
\operatorname{dim} j(\mathcal{H})=h^{0}(N)-h^{1}(N)=\chi(N)=\operatorname{deg} N+2(1-g)=-K_{X} \cdot C=d .
$$

This concludes the proof.

Example. Let $X$ be a prime Fano threefold of genus $g \geq 3$. Then $-K_{X}$ is very ample and defines an embedding $X \subset \mathbb{P}^{g+1}$. Consider the family $\mathcal{H}_{\text {can }}$ of canonical curves on $X$, that is, codimension two linear sections of $X$. Then $\operatorname{dim} \mathcal{H}_{\text {can }}=\operatorname{dim} G(2, g+2)=2 g$, while these curves have anticanonical degree $-K_{X}^{3}=2 g-2<\operatorname{dim} \mathcal{H}_{c a n}$. The image of the map

$$
j: \mathcal{H}_{\text {can }} \longrightarrow \mathrm{Hilb}_{2 g-2} S
$$

is the family of canonical punctual schemes on $S$, defined again by codimension two linear sections of $S$. Its dimension is $\operatorname{dim} j\left(\mathcal{H}_{\text {can }}\right)=\operatorname{dim} G(2, g+1)=2 g-2$, as expected, and $j$ is a $\mathbb{P}^{2}$-fibration. Note that a canonical curve $C$ has normal bundle $N=\mathcal{O}_{C}(1) \oplus \mathcal{O}_{C}(1)=K_{C} \oplus K_{C}$, so clearly $h^{1}(N)=2$. The fact that $j\left(\mathcal{H}_{\text {can }}\right)$ is Lagrangian already appears in [Voi], Exemple 3 .

The proof of the previous statement also provides a criterion for the map $j$ to be immersive.

Corollary 2 Let $\mathcal{H}$ be as above, and let $C$ be a curve of the family parameterized by $\mathcal{H}$. Suppose that $C$ is a locally complete intersection in $X$, with normal bundle $N$. Then $j$ is an immersion at $C$ if and only if the evaluation map

$$
e v_{C \cap S}: H^{0}(N) \longrightarrow N \otimes_{\mathcal{O}_{X}} \mathcal{O}_{C \cap S}
$$

is injective.

In the next section this criterion will be applied to conics on prime Fano threefolds. 


\section{Fano surfaces of conics on prime Fano threefolds}

Let $X$ be a prime Fano threefold of genus $g$. It is well known that $X$ contains lines, but there is only a one dimensional family of lines on $X$, and in particular $X$ is not covered by lines, see $\S 4.2$ in IP. But $X$ is covered by conics, and the Fano scheme $F(X)$ of conics on $X$ appears as a fundamental object of study, ibid.

In this section we focus on $F(X)$, for $X$ a general prime Fano threefold, and we apply to conics the main result of the previous section.

\subsection{Conics on general prime Fano threefolds}

First recall that the genus $g$ of a smooth prime Fano threefold $X$ can be any integer between 2 and 12 , except 11 , see IP.

For $g=2$, the anticanonical divisor is base point free and defines $X$ as a double cover of $\mathbb{P}^{3}$ ramified along a general sextic hypersurface (a sextic double solid). For $g \geq 3,-K_{X}$ is base point free, see Theorem 2.4.5 in [I]. Moreover $-K_{X}$ is very ample, with only one exception: the double quadrics $X_{4}^{\prime}$ for which $g=3$ and $-K_{X}$ defines a double covering from $X$ to the smooth quadric threefold, see Proposition 4.1.11 in [IP. Notice that $X_{4}^{\prime}$ is not the general prime Fano threefold of genus $g=3$, regarded in the context of our main Theorem 10,

In all the other cases the smooth $X=X_{2 g-2}$ are complete intersections in homogeneous spaces, as follows from the works of Mukai (see Muk1, and also [P]). For the convenience of the reader we recall below, for each value of $g \geq 3$, the nature of $\Sigma$, its dimension $d$, and the multidegree $\delta$ of $X$ in $\Sigma$.

$\begin{array}{llll}g & \delta & \Sigma & d \\ 3 & 4 & \mathbb{P}^{4} & 4 \\ 4 & 2,3 & \mathbb{P}^{5} & 5 \\ 5 & 2,2,2 & \mathbb{P}^{6} & 6 \\ 6 & 2,1,1 & G(2,5) & 6 \\ 7 & 1,1,1,1,1,1,1 & O G(5,10) & 10 \\ 8 & 1,1,1,1,1 & G(2,6) & 8 \\ 9 & 1,1,1 & L G(3,6) & 6 \\ 10 & 1,1 & G_{2}^{a d} & 5\end{array}$

Here $G(r, d)$ (resp. $O G(r, d), L G(r, d))$ is the Grassmannian of $r$-dimensional subspaces (resp. isotropic subspaces) of a $d$-dimensional vector space (resp. endowed with a non degenerate symmetric of skew-symmetric bilinear form, where we consider only one connected component in the case of $O G(r, 2 r)) . G_{2}^{a d}$ denotes the adjoint variety of the exceptional group $G_{2}$, the closed $G_{2}$-orbit in the projectivized Lie algebra $\mathbb{P}_{\mathfrak{g}_{2}}$.

For any $2 \leq g \leq 12, g \neq 11$ the Fano scheme of conics $F(X)$ is known to be a smooth surface, and the normal bundle to a general conic $q \subset X$ is trivial, see [CV] for $g=2$, CMW for $g=3$, IM2 for $g=4$, and Proposition 4.2.5 in IP for $g \geq 5$.

For $g=12$, the Fano surface $F(X) \simeq \mathbb{P}^{2}$, see Remark 5.2.15 in IP.

For $g=10, F(X)$ is an Abelian surface. Since we could not find any reference for this fact, we include a proof of the following statement: 
Proposition 3 For $X$ a general Fano threefold of genus 10, the Fano surface $F(X)$ and the intermediate Jacobian $J(X)$ are isomorphic.

Proof. We first prove that $F(X)$ is an Abelian surface. The idea to prove this is to construct an Abelian fibration on the Hilbert square of a general K3 surface $S$ of genus 10. The existence of this Abelian fibration is known by [HT] (see also [Saw] for a more general treatment of Abelian fibrations on Hilbert powers of K3 surfaces).

The general such $S$ is a hyperplane section of the general Fano threefold $X$ of genus 10 . Following Mukai, $S=\mathbb{P}_{S}^{10} \cap G_{2}^{a d}$ is a codimension three linear section of the adjoint variety of $G_{2}$. Also the general $X_{t}$ containing $S$ as a hyperplane section is a general linear section of $\Sigma$ by a codimension 2 subspace $\mathbb{P}_{t}^{11}$ containing $\mathbb{P}^{10}$. Let $\mathbb{P}^{2}$ be the family of all $\mathbb{P}_{t}^{10}$ 's containing $\mathbb{P}_{S}^{10}$. We define $\mathcal{F}(S)$ as the family of all conics that lie on the threefolds $X_{t}, t \in \mathbb{P}^{2}$. It can be seen that: (a) the 4-fold family $\mathcal{F}(S)$ is a disjoint union of the surfaces $F\left(X_{t}\right), t \in \mathbb{P}^{2}$ (see Lemma 7 below); (b) the intersection map $j: \mathcal{F}(S) \rightarrow \mathrm{Hilb}_{2} S, q \mapsto q \cap S$ defines an isomorphism between $\mathcal{F}(S)$ and $\mathrm{Hilb}_{2} S$. By (a), the family $\mathcal{F}(S)$ has a natural regular surjective map $f: \mathcal{F}(S) \rightarrow \mathbb{P}^{2}$ sending the conic $q \in \mathcal{F}(S)$ to the unique $t \in \mathbb{P}^{2}$ such that $q \in F\left(X_{t}\right)$. By (b), the map $f$ defines a regular surjective map $f_{o}: \mathrm{Hilb}_{2} S \rightarrow \mathbb{P}^{2}$. By the theorem of Matsushita (see [Msh), the general fiber $f_{o}^{-1}(t)$ of $f_{o}$ is an Abelian surface in $\mathrm{Hilb}_{2} S$. Since by construction the general fiber $f_{o}^{-1}(t) \simeq f^{-1}(t)$ is isomorphic to $F\left(X_{t}\right)$, identified with the family of conics on the general Fano 3 -fold $X_{t} \supset S$ then $F\left(X_{t}\right)$ is an Abelian surface.

The isomorphism of the Abelian surface $F(X)$ with the intermediate Jacobian of $J(X)$ can be derived on the base of the birational geometry of $X$ as follows.

Let $\ell \subset X$ be a line. By Theorem 4.3.3(vii) of [IP], the double projection $\pi_{\ell}$ from $\ell$ defines a birational isomorphism from $X$ to a 3 -fold quadric $Q$. The double projection $\pi_{\ell}$ contracts the family of conics intersecting $\ell$ to a smooth curve $\Gamma \subset Q$ of genus 2 and degree 7 ; therefore the intermediate Jacobian $J(X)$ is isomorphic to the Jacobian $J(\Gamma)$ of $\Gamma$.

By Matsusaka's criterion the Abelian surface $F(X)$ will be isomorphic to $J(\Gamma)$ if we can find on $F(X)$ a curve $C \simeq \Gamma$ with self-intersection number $C^{2}=2$, see Msk. We shall show that such a curve is the base $F_{\ell} \subset F(X)$ of the family of conics on $X$ intersecting $\ell$. First, by the preceding $F_{\ell} \simeq \Gamma$. Next, the base of the family $G(X)$ of lines on $X$ is a smooth irreducible curve (of genus 10), see Theorem 4.2.7 in IP. Therefore all the curves $F_{\ell}, \ell \in G(X)$ are algebraically equivalent to each other on the surface $F(X)$. So $F_{\ell}^{2}=F_{\ell} \cdot F_{m}$ for any other line $m$ on $X$. If $m \subset X$ is general then the intersection number $F_{\ell} \cdot F_{m}$ is the number of conics $q \subset X$ that intersect both $\ell$ and $m$. But according to Theorem 4.3.3 (vii) of $\underline{I P}$, the conics $q \in F_{\ell}$ sweep out a quadratic section $D_{\ell}$ of $X$. So $D_{\ell}$ intersects the line $m$ at two points, and therefore $F_{\ell} \cdot F_{m}=D_{\ell} \cdot m=2$.

For $g=9, F(X)$ is a ruled surface with base a smooth plane quartic $C_{X}$ such that $J(X) \simeq$ $J\left(C_{X}\right)$, see [li].

For $g=8, F(X)$ is isomorphic to the Fano surface of lines on the unique smooth cubic threefold $Y_{X}$ birational to $X$, IM1. The Fano surfaces of lines on cubic threefolds have been studied in deep by Clemens and Griffiths, CG.

For $g=7, F(X)$ is isomorphic to the symmetric square $S y m^{2} C_{X}$ of a genus 7 curve $C_{X}$, such that $J(X)=J\left(C_{X}\right)$, see IMr or $\mathrm{Kuz}$.

For $g=6$, the Fano surface $F(X)$ is smooth by $\log$, Proposition 0.1. 
For $g=3,4,5$, the Fano threefold $X$ is a complete intersection. This allows to describe $F(X)$ as the zero-locus of a section of some globally generated vector bundle; in particular $F(X)$ is smooth for $X$ general, see [CMW] for $g=3$, [M2 for $g=4$, Wel] and [PB] for $g=5$.

Finally, the smoothness of $F(X)$ for $g=2$ is proved by Ceresa and Verra, see Proposition 1.14 in $\mathrm{CV}$.

We compile some numerical data for the Fano surface $F=F(X)$ of a general prime Fano threefold $X$ of genus $g$.

$\begin{array}{ccccccc}g & c_{2} & c_{1}^{2} & p_{a} & \nu & q & F \\ 12 & 3 & 9 & 0 & 6 & 0 & \mathbb{P}^{2} \\ 10 & 0 & 0 & -1 & 9 & 2 & \text { Abelian } \\ 9 & -8 & -16 & -3 & 12 & 3 & \text { ruled } \\ 8 & 27 & 45 & 5 & 16 & 5 & \\ 7 & 66 & 114 & 14 & 24 & 7 & \text { Sym }^{2} C \\ 6 & 384 & 720 & 91 & 39 & 10 & \\ 5 & 1760 & 3376 & 427 & 148 & 14 & \\ 4 & 11961 & 23355 & 2942 & 318 & 20 & \\ 3 & 172704 & 341040 & 42811 & 972 & 30 & \end{array}$

We denoted by $\nu$ the number of conics through a general point of $X$. For the value of $\nu$ for $g \geq 8$, see the table (2.8.1) in Tak]. In the cases $g=7$ and $g=6$, the numbers $\nu=24$ and $\nu=39$ can be computed by the same approach as in [Tak], see e.g. Theorem 4.5.8 in [P]. The computation for $g=3$ is done in CMW, and the case $g=4$ is in IM2. The invariants in genus 5 are in Wel], except for the computation of $\nu$ which can be obtained with the same techniques as for $g=3,4$.

For $g=2$ (as well for all the other $g$ ) for the proof of Theorem 10 we shall need in fact only the known by $[\mathrm{CV}]$ invariant $q=52$. Beware that in the case $g=6$ the Fano surface $F(X)$ is the blow-up of a point on a smooth surface $F$; the table gives the invariants of $F$, see $\log$.

\subsection{Intersecting conics with a K3 surface}

Let $S \subset X$ be a K3 surface, defined as a general anticanonical section. Then the image of the natural map $j: F(X) \rightarrow \mathrm{Hilb}_{2} S$ is a possibly singular Lagrangian surface. In fact $j$ can be an embedding only if $g \geq 6$. Our precise result is the following:

Proposition 4 Let $X$ be a general Fano threefold of genus $g, S$ a general hyperplane section. Suppose that the curve $\Gamma(X)$ of lines on $X$ is smooth, and that the Fano surface $F(X)$ of conics on $X$ is also smooth. Then:

1. $j: F(X) \longrightarrow \mathrm{Hilb}_{2} \mathrm{~S}$ is an immersion.

2. $j$ is injective for $g \geq 7$ (but not for $g \leq 6$ ).

Proof. The injectivity of $j$ is equivalent to the non-existence of bisecant conics meeting along $S$. For $g \geq 7$ this was observed in [Voi], Section 2. For $g \leq 6$ this is no longer true, see the Remark below. 
Nevertheless we can check that $j$ is always immersive by applying the criterion of Corollary 2. Let $q \in F(X)$ be a smooth conic. Since $F(X)$ is smooth at $q$ and the normal bundle $N$ of $q$ in $X$ has trivial determinant, we must have $N=\mathcal{O}_{q} \oplus \mathcal{O}_{q}$ or $N=\mathcal{O}_{q}(1) \oplus \mathcal{O}_{q}(-1)$. In both cases the evaluation map

$$
e v_{q \cap S}: H^{0}(N) \longrightarrow N \otimes_{\mathcal{O}_{X}} \mathcal{O}_{q \cap S}
$$

is injective, were $q \cap S$ the union of two distinct points or the tangent direction to $q$ at some point.

Now we consider the case of a singular conic $q=\ell \cup m$, with vertex $p=\ell \cap m$. Since $q$ is a locally complete intersection, its normal bundle $N=N_{q / X}=\operatorname{Hom}\left(I_{q} / I_{q}^{2}, \mathcal{O}_{q}\right)$ is a locally free $\mathcal{O}_{q}$-module of rank two. Twisting the exact sequence $0 \rightarrow \mathcal{O}_{q} \rightarrow \mathcal{O}_{\ell} \oplus \mathcal{O}_{m} \rightarrow \mathcal{O}_{p} \rightarrow 0$ by $N$, we get

$$
0 \rightarrow N \rightarrow N(\ell) \oplus N(m) \rightarrow N_{p} \rightarrow 0,
$$

where $N(\ell)=N \otimes_{\mathcal{O}_{q}} \mathcal{O}_{\ell}$ is a locally-free $\mathcal{O}_{\ell}$-module. Note that since $I_{q} \supset I_{\ell} I_{m}, I_{m} N$ is also a $\mathcal{O}_{\ell}$-module, again locally free of rank two. In fact $I_{m} N=N(\ell)(-p)$, and we have a commutative diagram

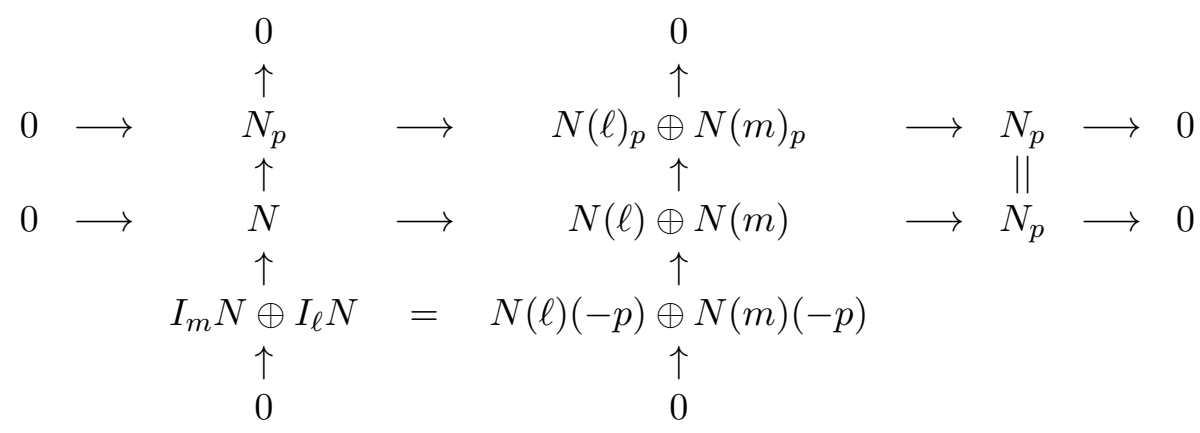

Beware that $I_{m} N$ is not isomorphic with $N_{\ell}$, the normal bundle to $\ell$, although these two sheaves are obviously isomorphic outside $p$. In fact, there is an exact sequence

$$
0 \longrightarrow I_{m} N \longrightarrow N_{\ell} \longrightarrow N_{\ell, p} / \operatorname{Im} T_{p} m=\mathcal{O}_{p} \rightarrow 0 .
$$

For $X$ general, the curve $\Gamma(X)$ of lines on $X$ is smooth, hence $N_{\ell} \simeq \mathcal{O}_{\ell} \oplus \mathcal{O}_{\ell}(-1)$. So there are two possibilities: either $I_{m} N \simeq \mathcal{O}_{\ell}(-1) \oplus \mathcal{O}_{\ell}(-1)$ or $I_{m} N \simeq \mathcal{O}_{\ell} \oplus \mathcal{O}_{\ell}(-2)$, and the latter case occurs if and only if the map

$$
H^{0}\left(N_{\ell}\right) \longrightarrow N_{\ell, p} / \operatorname{Im} T_{p} m
$$

is not injective. Anyway, we get three possibilities:

1. $N(\ell)$ and $N(m)$ are both trivial, hence $N$ itself is trivial;

2. $N_{\ell}$ is trivial but $N(m)=\mathcal{O}_{m}(1) \oplus \mathcal{O}_{m}(-1)$ (or the opposite): then $H^{0}(N) \simeq H^{0}(N(m)$ ), and each section is defined on restriction to $\ell$ by its value at $p$;

3. $N(\ell)=\mathcal{O}_{\ell}(1) \oplus \mathcal{O}_{\ell}(-1)$ and $N(m)=\mathcal{O}_{m}(1) \oplus \mathcal{O}_{m}(-1)$. Note this does not imply that $N=\mathcal{O}_{q}(1) \oplus \mathcal{O}_{q}(-1)$ : this is true only if the two distinguished directions in $N_{p}$ fit together, which is in fact excluded by the condition that $h^{0}(N)=2$.

Now we need to check that the evaluation map $e v_{x} \oplus e v_{y}: H^{0}(N) \longrightarrow N_{x} \oplus N_{y}$ is injective, if $x=\ell \cap S$ and $y=m \cap S$ are distinct; if they coincide, we want $e v_{x}^{1}: H^{0}(N) \longrightarrow N \otimes \mathcal{O}_{q} / \mathcal{M}_{x}^{2}$ to be injective. For the three possibilities above this is a straightforward verification. 
Corollary 5 For $X$ a general prime Fano threefold of genus $g \geq 7$, and $S$ a general hyperplane section, $j(F(X))$ is a smooth Lagrangian surface in $\mathrm{Hilb}_{2} \mathrm{~S}$.

Remark. For $g=6$ recall that $X$ is a complete intersection of multidegree $\delta=(2,1,1)$ in $G(2,5)$. For any conic $q$ in $X$, there is a unique hyperplane $H$ in $\mathbb{C}^{5}$ such that $q$ is contained in $G(2, H)$. Since the intersection of $X$ with $G(2, H)$ has degree four, it must be the union of $q$ with an involutive conic $q^{\prime}$, which is bisecant to $q$. By an obvious dimension count, there must exist a finite number of conics $q$ on $X$, whose two common points with the involutive conic $q^{\prime}$ lie on $S$ - and in this case $j(q)=j\left(q^{\prime}\right)$. This shows that $j(F(X))$ is not smooth but has finitely many double points.

\subsection{Getting back from $j(F(X))$ to $X$}

It is not true in general that $X$ is uniquely determined by the surface $F(X)$. For example, the Fano surface of the general $X_{22}$ is isomorphic to $\mathbb{P}^{2}$, but the Fano 3 -folds $X_{22}$ have 6 moduli, see e.g. Corollary 1.2 in Sch. Similarly, one can see that the Fano surface of the general $X_{18}$ is the Jacobian of the general genus 2 curve and has 3 moduli, while $X_{18}$ has 10 moduli.

Here we consider a slightly different question: does $j(F(X)) \subset \mathrm{Hilb}_{2} S$ define $X$ uniquely among those Fano threefolds that contain $S$ ?

Theorem 6 For $7 \leq g \leq 10$, a Fano threefold $X$ containing a fixed K3 surface $S$ as a hyperplane section, is uniquely determined by the Lagrangian surface $j(F(X)) \subset \mathrm{Hilb}_{2} \mathrm{~S}$.

We do not know whether this statement is still true for $g \leq 6$.

Proof. We want to recover $X \supset S$ from the embedded Lagrangian surface $j(F(X)) \subset H_{i l b_{2}}(S)$. By [Muk1], $S$ and $X$ have linear embeddings in a rational homogeneous variety $\Sigma=G / P$ of some simple adjoint Lie group $G$, and this embedding is unique up to the action of $G$. Therefore we may assume that $S \subset X \subset G / P$.

Now we have to consider separately the different values of $g$.

$g=10$. Here $G=G_{2}$ and $G / P$ is the adjoint $G_{2}$-variety, the variety parametrizing highest root spaces in the projectivized Lie algebra $\mathbb{P} \mathfrak{g}_{2}$.

Lemma 7 Consider two general points $x, y$ in the adjoint variety $G_{2} / P$. Then there is a unique conic in $G_{2} / P$ passing through $x$ and $y$.

Proof of the lemma. First, there exists at least one conic in $G_{2} / P$ through $x$ and $y$. Indeed, the Lie subalgebra of $\mathfrak{g}_{2}$ generated by $x$ and $y$ is a copy of $\mathfrak{s l}_{2}$, and generates in $G_{2}$ a copy of $S L_{2}$. The $S L_{2}$-orbits of $x$ and $y$ have the same closure, which is a conic passing through $x$ and $y$ in $G_{2} / P$. (To check this, choose a maximal torus in $\mathfrak{g}$ with respect to which $x$ and $y$ are highest and lowest root vectors.)

On the other hand, the group $G_{2}$, being the automorphism group of the (complexified) algebra of octonions, has a minimal representation of dimension 7 on the imaginary octonions. Since it preserves the norm, we deduce in particular that the adjoint variety $G_{2} / P$ is a subvariety of $G_{Q}(2,7)$, the Grassmannian of lines in a smooth quadric $Q \subset P^{6}$. But through two general points of a Grassmannian of lines in a smooth quadric there exists a unique conic, which can be constructed as follows. The two points $x$ and $y$ represent two skew lines $\ell_{x}, \ell_{y} \subset Q$. These two lines generate $P_{x, y}^{3}=\operatorname{Span}\left(\ell_{x} \cup \ell_{y}\right)$ intersecting $Q$ along a quadric surface $S_{x, y}$. One 
of the two rulings of this surface contains $\ell_{x}, \ell_{y}$; it defines a conic $q$ in $G_{Q}(2,7)$ passing through $x$ and $y$.

From $j(F(X)) \subset \mathrm{Hilb}_{2} S$ we can therefore recover the Fano surface $F(X)$, and then the threefold $X$ itself since it is swept out by the conics it contains.

$g=9$. Here $G=S p_{6}$ and $G / P=L G(3,6)$, the Lagrangian Grassmannian.

Recall from [li, IR] that a conic $q \subset L G(3,6)=L G\left(\mathbb{P}^{2}, \mathbb{P}^{5}\right)$ has a vertex-point $v(q) \in \mathbb{P}^{5}$, defined as the unique intersection point of the Lagrangian planes $\mathbb{P}_{t}^{2}, t \in q$.

A general conic $q \subset X$ is smooth and intersects $S$ at two different points $x, y$, from which the vertex can be recovered as $v(q)=\mathbb{P}_{x}^{2} \cap \mathbb{P}_{y}^{2}$. In particular, $j(F(X))$ determines the vertex surface $v(F(X))$. By Ili], for a general Fano threefold $X$ of genus 8 the vertex image $v(F(X)) \subset \mathbb{P}^{5}$ is a ruled surface isomorphic to $F(X)$. By $\llbracket$ IR, Proposition 2.8.5, $X$ can be recovered as the set of projective planes in $\mathbb{P}^{5}$ that cut the vertex surface along a finite scheme of length 12 .

$g=8$. Here $G=P S L_{6}$ and $G / P=G(2,6)$ is the Grassmannian of (projective) lines.

If $S \subset X \subset G(2,6) \subset \mathbb{P}\left(\wedge^{2} \mathbb{C}^{6}\right)$, let $L_{X} \subset L_{S}$ be the orthogonal in $\mathbb{P}\left(\wedge^{2} \mathbb{C}^{6}\right)^{*}$ of the linear spans of $S$ and $X$. They are projective spaces of dimension four and five respectively. We want to show that, the embedding $j(F(X)) \subset H_{i l b} S$ being given, the hyperplane $L_{X}$ of $L_{S}$ is uniquely determined.

A conic $q$ in $G(2,6)$ whose linear span is not contained in $G(2,6)$, defines a unique 4 -space $V \subset \mathbb{C}^{6}$ such that $q \subset G(2, V)$. Moreover, $q$ can be obtained as the intersection of the four dimensional quadric $G(2, V) \subset \mathbb{P} \wedge^{2} V$ with some projective plane. In particular, $G(2, V)$ contains some conic of $X$ if and only if $\mathbb{P} \wedge^{2} V$ meets $L_{X}^{\perp}$ along a projective space of dimension two at least. We thus consider on $G=G(4,6)$ the tautological rank four vector bundle $T$. By restriction of skew-symmetric forms, we have morphisms of vector bundles

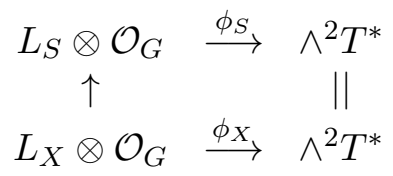

Since $\phi_{X}$ is a general morphism from $\mathcal{O}_{G}^{\oplus 5}$ to the rank six vector bundle $\wedge^{2} T^{*}$, its degeneracy locus $\Sigma(X)=D_{3}\left(\phi_{X}\right)$ has codimension $(5-3)(6-3)=6$ and is singular exactly along $D_{2}\left(\phi_{X}\right)$, which has codimension $(5-2)(6-2)=12>8$. So $\Sigma(X)$ is a smooth surface, and since it is in bijective correspondence with $F(X)$ these two surfaces must be isomorphic.

On the other hand, the degeneracy locus $\Sigma(S)=D_{4}\left(\phi_{S}\right)$ has codimension $(6-4)(6-4)=4$ and is singular exactly along $D_{3}\left(\phi_{S}\right)$, which has codimension $(6-3)(6-3)=9>8$. So $\Sigma(S)$ is a smooth four dimensional subvariety of $G(4,6)$. On $\Sigma(S)$ the morphism $\phi_{S}$ has constant rank four, so in the exact sequence

$$
0 \longrightarrow \operatorname{Ker} \phi_{S} \longrightarrow L_{S} \otimes \mathcal{O}_{G} \stackrel{\phi_{S}}{\longrightarrow} \wedge^{2} T^{*} \longrightarrow \operatorname{Coker} \phi_{S} \longrightarrow 0
$$

$\operatorname{Ker} \phi_{S}$ and $\operatorname{Coker} \phi_{S}$ are rank two vector bundles. Note that if we take determinants in this sequence, we get

$$
\operatorname{det}\left(\operatorname{Coker}_{S}\right) \operatorname{det}\left(\operatorname{Ker} \phi_{S}\right)^{-1}=\operatorname{det}\left(\wedge^{2} T^{*}\right)=\mathcal{O}_{G}(3) .
$$

Now the normal bundle of $\Sigma(S)$ in $G$ is naturally identified with $\operatorname{Hom}\left(\operatorname{Ker} \phi_{S}, \operatorname{Coker} \phi_{S}\right)$, whose determinant is $\operatorname{det}\left(\operatorname{Coker} \phi_{S}\right)^{2} \operatorname{det}\left(\operatorname{Ker} \phi_{S}\right)^{-2}=\mathcal{O}_{G}(6)=K_{G}^{-1}$. Therefore $\Sigma(S)$ has trivial 
canonical bundle. Now the natural map $\Sigma(S) \rightarrow \mathrm{Hilb}_{2} S$ is finite, hence étale, hence an isomorphism since $\mathrm{Hilb}_{2} \mathrm{~S}$ is simply connected. We conclude that the embedding $j(F(X)) \subset \mathrm{Hilb}_{2} S$ is the same as the embedding $\Sigma(X) \subset \Sigma(S)$.

But from $L_{S}$, its degeneracy locus $\Sigma(S)$ and the embedding $\Sigma(X) \subset \Sigma(S)$, the hyperplane $L_{X}$ of $L_{S}$ can be recovered as follows. At any point $x \in \Sigma(X)$, the morphisms $\phi_{S}$ ans $\phi_{X}$ have the same kernel, which in particular is contained in $L_{X}$. So we must have

$$
L_{X}=\operatorname{Span}\left(\operatorname{Ker} \phi_{S, x}, x \in \Sigma(X)\right) \subset L_{S} .
$$

And then of course $X$ can be recovered as $X=G(2,6) \cap L_{X}^{\perp}$.

$g=7$. Here $G=\operatorname{Spin}_{10}$ and $G / P=\Sigma \subset \mathbb{P} \Delta_{+}$is the spinor variety, naturally embedded in the projectivization of a half-spin representation.

If $S \subset X \subset \Sigma \subset \mathbb{P} \Delta_{+}$, let $L_{X} \subset L_{S}$ be the orthogonal in $\mathbb{P} \Delta_{-}$of the linear spans of $S$ and $X$. They are projective spaces of dimension seven and eight respectively. Again we want to show that, the embedding $j(F(X)) \subset \mathrm{Hilb}_{2} S$ being given, the hyperplane $L_{X}$ of $L_{S}$ is uniquely determined.

A conic $q$ in $\Sigma$ whose linear span is not contained in $\Sigma$, has a vertex $v=v(q) \in \mathbb{P}^{9}$. The set of isotropic planes in $\Sigma$ passing through $v$ is a copy of the spinor variety of $\operatorname{Spin}_{8}$. By triality, this spinor variety is a six dimensional quadric $Q_{v} \subset \Sigma$. Moreover, $q$ can be obtained as the intersection of $Q_{v}$ with some projective plane. In particular, $Q_{v}$ contains some conic of $X$ if and only if its linear space $P_{v}$ meets $L_{X}^{\perp}$ along a projective space of dimension two at least.

This linear space $P_{v}$ defines on the eight-dimensional quadric $\mathbb{Q}^{8}$ in $\mathbb{P}^{9}$ a spinor bundle $S$ of rank eight Ott]. We get on $\mathbb{Q}^{8}$ the morphisms of vector bundles

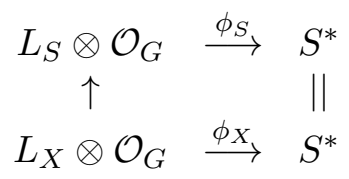

Exactly as in genus eight, the degeneracy locus $\Sigma(X)=D_{3}\left(\phi_{X}\right)$ is a smooth surface isomorphic with $F(X)$. Also the degeneracy locus $\Sigma(S)=D_{2}\left(\phi_{S}\right)$ is a smooth four dimensional variety with trivial canonical bundle (because $\operatorname{det} S=\mathcal{O}_{Q}(4)$ ), in fact isomorphic with $\mathrm{Hilb}_{2} S$. And from the embedding $\Sigma(X) \subset \Sigma(S)$, which is the same as $j(F(X)) \subset H i l b_{2} S$, we can recover $L_{X}$ in $L_{S}$ as before.

This concludes the proof.

\section{Fano-Integrable Systems}

Thanks to the main results of the previous section, we are now in position to apply the DonagiMarkman construction [DM]. The upshot will be new Lagrangian fibrations over families of prime Fano threefolds with a fixed "boundary", whose fibers are intermediate Jacobians.

\subsection{The Abel-Jacobi isomorphism for conics on prime Fano's}

The intermediate Jacobian $J(X)$ a prime Fano threefold of genus $g$ is an Abelian variety of dimension $h^{1,2}(X)=b_{3}(X) / 2$ (see CG for the definition and general properties of intermediate Jacobians). 
This dimension can be computed case by case. Note that for $7 \leq g \leq 10$, we can use the fact that $X$ is a linear section of a $d$-dimensional homogeneous space $\Sigma \subset \mathbb{P}^{d+g-2}$. After twisting the normal exact sequence by $K_{X}=\mathcal{O}_{X}(-1)$, we get

$$
0 \longrightarrow \Omega_{X}^{2} \longrightarrow T \Sigma \otimes \mathcal{O}_{X}(-1) \longrightarrow \mathcal{O}_{X}^{\oplus d-3} \longrightarrow 0 .
$$

With the help of the Bott-Borel-Weil theorem one can check that $H^{i}\left(\Sigma, T \Sigma \otimes \mathcal{O}_{\Sigma}(-i-1)\right)=$ $H^{i+1}\left(\Sigma, T \Sigma \otimes \mathcal{O}_{\Sigma}(-i-1)\right)=0$ for $0 \leq i \leq d-3$. Using the Koszul resolution of $\mathcal{O}_{X}$ this implies that $H^{0}\left(X, T \Sigma \otimes \mathcal{O}_{X}(-1)\right)=H^{1}\left(X, T \Sigma \otimes \mathcal{O}_{X}(-1)\right)=0$. Hence the isomorphism

$$
H^{1}\left(\Omega_{X}^{2}\right)=\mathbb{C}^{d-3}=L_{X},
$$

if we denote by $L_{X}$ the space of linear forms defining $X$ in $\Sigma$.

Note also that if $X$ has no automorphism, which is the general case, we get an exact sequence

$$
0 \longrightarrow H^{0}\left(X, T \Sigma_{\mid X}\right) \simeq \mathfrak{g} \longrightarrow L_{X} \otimes L_{X}^{\perp} \longrightarrow H^{1}(X, T X) \longrightarrow 0,
$$

where $\mathfrak{g}$ denotes the Lie algebra of the Lie group $G$; hence $h^{1}(T X)=(g+2)(d-3)-\operatorname{dim} G$. The analogue sequence for $S$ is

$$
0 \longrightarrow H^{0}\left(S, T \Sigma_{\mid S}\right) \simeq \mathfrak{g} \longrightarrow L_{S} \otimes L_{S}^{\perp} \longrightarrow H^{1}(S, T S) \longrightarrow \mathbb{C} \longrightarrow 0 .
$$

This gives $h^{1}(T S)-1=(g+1)(d-2)-\operatorname{dim} G=19$, and thus

$$
h^{1}(T X)=15+d-g=h^{1}\left(\Omega_{X}^{2}\right)+18-g .
$$

A basic property of the intermediate Jacobian is that for any family $\mathcal{C} \rightarrow F$ of curves in $X$, parameterized by a smooth base $F$, there is an induced Abel-Jacobi mapping $F \rightarrow J(X)$.

Theorem 8 Let $X$ be a general prime Fano threefold of genus $g$, with a smooth surface of conics $F(X)$. Then the Abel-Jacobi mapping $F(X) \rightarrow J(X)$ induces an isomorphism

$$
\operatorname{AlbF}(X) \simeq J(X) .
$$

Proof. For most values of $g$, this Abel-Jacobi isomorphism is already directly or indirectly known, so below we refer to the original references, interpret some other in the required context, and complete the argument for the genera $g=10$ and 6 missing in the known to us literature.

The case $g=12$ is trivial since then $h^{1,2}(X)=0$ and $F(X)$ is isomorphic to $\mathbb{P}^{2}$, see $\S 3.1$.

For $g=10$, by Proposition 3 the Fano surface $F(X)$ is isomorphic to $J(X)$ so in this case there is already nothing more to prove.

For $g=9, F(X)$ is a ruled surface over the orthogonal plane quartic curve $C_{X}$, therefore $\operatorname{AlbF}(X)=J\left(C_{X}\right)$, see $\S 3.1$. The fact that $J\left(C_{X}\right) \simeq J(X)$ is proved in [R, Corollary 2.8.10.

For $g=8, F(X)$ is isomorphic to the Fano surface $F(Y)$ of lines in the orthogonal cubic threefold $Y$, see $\S 3.1$. The intermediate Jacobians of $X$ and $Y$ are naturally isomorphic. The Fano surface $F(X)$ embeds in $J(X), F(Y)$ embeds in $J(Y)$, and their images coincide up to a multiplication by -1 (IM1, Proposition 4.6). So the Abel-Jacobi isomorphism for $F(X)$ follows from the corresponding statement for $F(Y)$, which is due to Clemens and Griffiths [CG].

For $g=7, F(X)$ is isomorphic to $S y m^{2} C_{X}$, where $C_{X}$ is the $\operatorname{Spin}_{10}$-orthogonal curve to $X$, see $\S 3.1$. From where $\operatorname{AlbF}(X) \simeq J\left(C_{X}\right)$. As known by $\S 8$ of Muk2 the Jacobian $J\left(C_{X}\right)$ is isomorphic to $J(X)$, see also [IMr]. 
For $g=3$, the Abel-Jacobi isomorphism for $F(X)$ has been proved by Letizia, by applying a degeneration technique originating from Clemens, see [Let]. After that, the same method had been used by Ceresa and Verra (see [CV]), Picco Botta (see [PB] and in our recent paper [M2] to prove the same result correspondingly for $g=2,5$ and 4 .

The case $g=6$ has been treated by Logachev (before the appearance of the paper [Let]) with the conclusion that $\operatorname{AlbF}(X) \rightarrow J(X)$ in an isogeny, by following an earlier argument of Welters proving the same isogeny for the case $g=5$, see Theorem 6.12 in Log and Wel. In fact, the Clemens-Letizia method applies also in the case $g=6$ to conclude that the Abel-Jacobi map is an isomorphism also for $g=6$. All the necessary conditions for this (a precise description of the Fano surface for a general nodal Fano threefold) are provided in Section 5 of Log.

\subsection{The intermediate Jacobian integrable systems for prime Fano's}

Consider a projective symplectic variety $Y$ of dimension $2 n$, a smooth variety $L$ of dimension $n$ and a Lagrangian immersion $f: L \rightarrow Y$. Then:

1. If $\mathcal{L} \rightarrow \mathcal{H}$ is a local semi-universal deformation of $f$, with fixed target $Y$, then $\mathcal{H}=$ $\operatorname{Def}(f / Y)$ is smooth ( $\mathbb{R a n}$, Corollary 3.4).

2. The tangent space to $\mathcal{H}$ at $L$ is

$$
T_{f} \mathcal{H}=H^{0}\left(L, N_{f}\right) \simeq H^{0}\left(L, \Omega_{L}^{1}\right),
$$

where the bundle $N_{f}$ is defined by the exact sequence $0 \rightarrow T L \rightarrow f^{*} T Y \rightarrow N_{f} \rightarrow 0$; indeed the fact that $f$ is Lagrangian yields an isomorphism $N_{f} \simeq \Omega_{L}^{1}$.

3. Any small deformation of $f$ remains a Lagrangian immersion in $Y$, see [Doi].

4. The relative Picard bundle $P i c^{0} \mathcal{L} \longrightarrow \mathcal{H}$ is a Lagrangian fibration (or an algebraic completely integrable Hamiltonian system), see [DM]. In fact Donagi and Markman only treat the case where $j$ is an embedding, that is, they consider the deformations of a smooth Lagrangian subvariety in a symplectic manifold. Nevertheless, their proof extends verbatim to the setting of Lagrangian immersions.

Let $X$ be a general prime Fano threefold of genus $g$ and $S$ a K3 surface in $X$. We can apply the results above to $f=j: L=F(X) \rightarrow Y=H_{i l b} S$, since we have proved in Proposition 4 that $j$ is indeed a Lagrangian immersion. We get that the family $\operatorname{Pic}^{0} \mathcal{L}$ over $\operatorname{Def}\left(j / \mathrm{Hilb}_{2} S\right)$ is a Lagrangian fibration.

Now we want to deform $X$ with $S$ fixed. The tangent space to the corresponding deformation functor is $H^{1}\left(X, T X \otimes \mathcal{I}_{S}\right)$ (see [Be3]). Since $\mathcal{I}_{S}=\mathcal{O}_{X}(-S)=K_{X}$, this is also $H^{1}\left(\Omega_{X}^{2}\right)$, the universal covering of $J(X)$.

We begin with a simple observation.

Proposition 9 There exists a semi-universal algebraic family $\mathcal{H}_{S}$ parameterizing deformations of $X$ containing $S$.

Proof. Suppose for example that $7 \leq g \leq 10$ (in which case $j$ is an embedding). Recall that by Mukai's theorem, $S$ can be described as a linear section $S=\Sigma \cap L \frac{\perp}{S}$ of a $d$-dimensional homogeneous space $\Sigma$ by a projective space of codimension $d-2$. The set of prime Fano 
threefolds $X$ containing as an anticanonical section, is then identified with an open subset of the orthogonal projective space $L_{S} \simeq \mathbb{P}^{d-3}$.

In the complete intersection case, consider for example the general quartic threefold $X \subset$ $\mathbb{P}^{4}$ admitting $S$ as a hyperplane section. The set of such $X$ 's is given by the set of quartic polynomials of the form $k=k_{S}+x_{0} c$, where $x_{0}$ is an equation of the hyperplane $H$ spanned by $S$ in $\mathbb{P}^{4}, k_{S}$ is an equation of $S$ in $H$, and $c$ is any cubic form. So the smooth quartic hypersurfaces of $\mathbb{P}^{4}$ containing $S$ are parameterized by an open subset of a 35 -dimensional affine space. Moreover the 5-dimensional subgroup $\Gamma_{H}$ of $G L_{5}$, acting trivially on $H$, acts on this affine space, and any linear subspace transverse to the orbit of $X$ defines a semi-universal, 30-dimensional, deformation of $X$ with $S$ fixed. The other cases are similar.

We have a natural map from the germ $\left(\mathcal{H}_{S}, \mathrm{X}\right)$ to $\operatorname{Def}\left(j / \mathrm{Hilb}_{2} S\right)$, whose differential

$$
H^{1}(X, T X(-S))=H^{1}\left(X, \Omega_{X}^{2}\right) \longrightarrow H^{0}\left(F(X), \Omega_{F(X)}^{1}\right)
$$

is an isomorphism when the Abel-Jacobi isomorphism does hold. In particular we can pull-back our Lagrangian fibration to $\mathcal{H}_{S}$, first locally, and then everything globalizes since the fibration is defined canonically - more precisely, we can pull it back to the open subset $\mathcal{H}_{S}^{0}$ on which $X$ is smooth, $F(X)$ is also smooth and the Abel-Jacobi isomorphism theorem does hold. But then over $\mathcal{H}_{S}^{0}, \operatorname{Pic} c^{0} F(X) \simeq A l b F(X)^{*} \simeq J(X)^{*} \simeq J(X)$, the intermediate Jacobian being self-dual. Since this isomorphism certainly holds in families, we finally deduce our main result:

Theorem 10 Let $S$ be a general K3 surface of genus $g$, with $2 \leq g \leq 10$. Let $\mathcal{X} \rightarrow \mathcal{H}_{S}$ be an algebraic semi-universal family of smooth Fano threefolds containing $S$.

Then the family of intermediate Jacobians $J(\mathcal{X}) \rightarrow \mathcal{H}_{S}$ is a Lagrangian fibration over a dense open subset $\mathcal{H}_{S}^{0}$ of $\mathcal{H}_{S}$.

For $g \geq 7$ the situation is even cleaner: one can take for $\mathcal{H}_{S}$ an open subset of the projective space $L_{S}$, and by Theorem 6 there is a birational isomorphism between $L_{S}$ and the component $\mathcal{H}$ of the Hilbert scheme of $\mathrm{Hilb}_{2} \mathrm{~S}$ parameterizing the Lagrangian surfaces $j F(X)$, for $X$ a general Fano threefold containing $S$.

\section{References}

[Be1] A. Beauville, Variétés Kählériennes dont la première classe de Chern est nulle, J. Differential Geom. 18, 755-782 (1983).

[Be2] A. Beauville, Systèmes hamiltoniens complètement intégrables associés aux surfaces K3, in Problems in the theory of surfaces and their classification (Cortona, 1988), 25-31, Sympos. Math. XXXII, Academic Press, London 1991.

[Be3] A. Beauville, Fano threefolds and K3 surfaces, The Fano Conference, 175-184, Univ. Torino, Turin 2004.

[BD] A. Beauville, R. Donagi, La variété des droites d'une hypersurface cubique de dimension 4, C. R. Acad. Sci., Paris, Sér. I, 301, 703-706 (1985).

[Bot] F. Bottacin, Poisson structures on Hilbert schemes of points on a surface and integrable systems, Manuscripta Math. 97, 517-527 (1998).

[CG] H. Clemens, Ph.A. Griffiths, The intermediate Jacobian of the cubic threefold, Annals of Math. 95, 281-356 (1972). 
[Col] A. Collino, The Abel-Jacobi isomorphism for the cubic fivefold, Pacific J. Math. 122 No.1, 43-55 (1986).

[CV] G. Ceresa, A. Verra, The Abel-Jacobi isomorphism for the sextic double solid, Pacific J. Math. 124 No.1, 85-105 (1986).

[CMW] A. Collino, J.P. Murre, G.E. Welters, On the family of conics lying on a quartic threefold, Rend. Sem. Mat. Univ. Politec. Torino 38 No.1, 151-181 (1980).

[DM] R. Donagi, E. Markman, Spectral covers, algebraically completely integrable, Hamiltonian systems, and moduli of bundles, in Francaviglia, M. (ed.) et al., Integrable systems and quantum groups, Lecture Notes in Math. 1620, 1-119 (1996).

[HT] B. Hassett, Yu. Tschinkel, Abelian fibrations and rational points on symmetric products, International J. Math. 11 No.9, 1163-1176 (2000).

[Ili] A. Iliev, The $\mathrm{Sp}_{3}$-Grassmannian and duality for prime Fano threefolds of genus 9, Manuscripta Math. 112 No.1, 29-53 (2003).

[IM1] A. Iliev, L. Manivel, Pfaffian lines and vector bundles on Fano threefolds of genus 8, e-print: math.AG/0504595, to appear in: J. Alg. Geom.

[IM2] A. Iliev, L. Manivel, Cubic hypersurfaces and integrable systems, e-print: math.AG/0605260

[IMr] A. Iliev, D. Markushevich, Elliptic curves and rank-2 vector bundles on the prime Fano threefold of genus 7, Advances in Geom. 4 No.3, 287-318 (2004).

[IR] A. Iliev, K. Ranestad, Geometry of the Lagrangian Grassmannian LG(3,6) with applications to Brill-Noether loci, Michigan Math. J. 53 No.2, 383-417 (2005).

[IP] V. Iskovskikh, Yu.G. Prokhorov, Fano varieties, in Algebraic Geometry V (A. N. Parsin, I. R. Shafarevich, Eds.), Encyclopaedia of Math. Sci. 47, Springer 1998.

[KLS] D. Kaledin, M. Lehn, Ch. Sorger, Singular symplectic moduli spaces, e-print: math.AG/0504202

[Kuz] A. Kuznetsov, Derived category of $V_{12}$ Fano threefolds, e-print: math.AG/0310008

[Let] M. Letizia, The Abel-Jacobi mapping for the quartic threefold, Inventiones Math. 75 No.3, 477-492 (1984).

[Log] D. Logachev, Fano threefolds of genus 6, e-print: math.AG/0407147.

[Msh] D. Matsushita, On fibre space structures of a projective irreducible symplectic manifold, Topology 38 No.1, 79-83 (1999); Addendum: Topology 40 No.2, 431-432 (2001).

[Msk] T. Matsusaka, On a characterization of a Jacobian variety, Mem. Coll. Sci., Univ. Kyoto, Ser.A 32, 1-19 (1959).

[Muk1] S. Mukai, Curves, K3 surfaces and Fano 3-folds of genus $\leq 10$, in Algebraic geometry and commutative algebra, Vol. I, 357-377, Kinokuniya, Tokyo 1988.

[Muk2] S. Mukai, Non-Abelian Brill-Noether theory and Fano 3-folds, Sugaku Expositions 14 No.2, 125-153 (2001).

[Ott] G. Ottaviani, Spinor bundles on quadrics, Trans. Amer. Math. Soc. 307 No.1, 301-316 (1988).

[PB] L. Picco Botta, On the intersection of three quadrics, J. Reine Angew. Math. 399, 188-207 (1989). 
[Ran] Z. Ran, Hodge theory and deformations of maps, Compositio Math. 97 No.3, 309-328 (1995).

[Saw] J. Sawon, Lagrangian fibrations on Hilbert schemes of points on K3 surfaces, e-print: math.AG/0509224

[Sch] F.-O. Schreyer, Geometry and algebra of prime Fano 3-folds of genus 12, Compositio Math. 127 No.3, 297-319 (2001).

[Tak] K. Takeuchi, Some birational maps of Fano 3-folds, Compositio Math. 71, 265-283 (1989).

[Tho] R. Thomas, Gauge theories on Calabi-Yau manifolds D. Phil. Thesis, University of Oxford, 1997; available on the homepage of R. Thomas: http://www.ma.ic.ac.uk/ rpwt/papers.html

[Tyu] A.N. Tyurin, Non-abelian analogues of Abel's theorem, Izv. Math. 65 No.1, 123-180 (2001).

[Voi] C. Voisin, Sur la stabilité des sous-variétés lagrangiennes des variétés symplectiques holomorphes, London Math. Soc. Lect. Note Ser. 179, 294-303 (1992).

[Wel] G.E. Welters, Abel-Jacobi isogenies for certain types of Fano threefolds, Mathematical Centre Tracts 141, Mathematisch Centrum, Amsterdam 1981.

\author{
Atanas Iliev \\ Institute of Mathematics \\ Bulgarian Academy of Sciences \\ Acad. G. Bonchev Str., bl.8 \\ 1113 Sofia, Bulgaria \\ e-mail: ailiev@math.bas.bg \\ Laurent Manivel \\ Institut Fourier \\ UMR 5582 (UJF-CNRS), BP 74 \\ 38402 St Martin d'Hères Cedex, France \\ e-mail: Laurent.Manivel@ujf-grenoble.fr
}

